\title{
Hypertriglyceridemic Pancreatitis Treated with Insulin Therapy: A Comparative Review of 34
} Cases

Faisal Inayat ${ }^{1}$, Fahad Zafar ${ }^{2}$, Asad S. Baig ${ }^{3}$, Najaf A. Chaudhry ${ }^{4}$, Aysha Aslam ${ }^{5}$, Zarak H. Khan ${ }^{6}$, Muhammad J. Iqbal ${ }^{1}$

1. Internal Medicine, Allama Iqbal Medical College, Lahore, PAK 2. Internal Medicine, King Edward Medical University, Lahore, PAK 3. Internal Medicine, Nawaz Sharif Medical College, Gujrat, PAK 4. Internal Medicine, Lahore Medical and Dental College, Lahore, PAK 5. Internal Medicine, Beth Israel Deaconess Medical Center, Boston, USA 6. Internal Medicine, St. Mary Mercy Hospital, Livonia, USA

Corresponding author: Faisal Inayat, faisalinayat@hotmail.com

\begin{abstract}
Although the clinical presentation of hypertriglyceridemic pancreatitis is usually similar to other forms of acute pancreatitis, it is frequently associated with increased clinical severity and rate of complications. Therefore, appropriate and timely management is of paramount importance in these patients. We performed a structured literature search of the medical databases PubMed and Google Scholar, using the terms "hypertriglyceridemia," "acute pancreatitis," "insulin," and "treatment." In this search, we identified 34 cases of hypertriglyceridemia-related pancreatitis available in the full-text form in English. The data on patients' characteristics, epidemiology, clinical features, comorbid conditions, and diagnostic modalities were collected and summarized. This review illustrates that the use of insulin therapy with close monitoring of blood glucose levels is safe. It can be considered as an important component of management in patients with hypertriglyceridemia-related pancreatitis, especially in a clinical setting without the availability of plasmapheresis. Randomized clinical trials are warranted to outline a generalized and efficient treatment regimen for hypertriglyceridemic pancreatitis.
\end{abstract}

Received 09/25/2018

Review began 10/01/2018 Review ended 10/18/2018 Published 10/27/2018

\section{(c) Copyright 2018}

Inayat et al. This is an open access article distributed under the terms of the Creative Commons Attribution License CC-BY 3.0., which permits unrestricted use, distribution, and reproduction in any medium, provided the original author and source are credited.
Categories: Gastroenterology

Keywords: hypertriglyceridemia, acute pancreatitis, insulin therapy, diagnosis, treatment, clinical outcomes

\section{Introduction And Background}

Acute pancreatitis is a common inflammatory condition of the pancreas with various underlying etiologies. Gallstones and alcohol are among the most common while drug-induced disease, autoimmune conditions, viral infections, trauma, and malignancy are less common causes [1-3]. However, relatively infrequent hypertriglyceridemia is one of the known etiologies of acute pancreatitis [4]. In patients with hypertriglyceridemic pancreatitis (HTGP), a pre-existing lipid abnormality has commonly been documented. According to prior research, the risk of acute pancreatitis increases significantly when serum hypertriglyceridemia exceeds 1,000 mg/dL (normal, 101-150 mg/dL) [5]. The concomitant presence of single or multiple secondary precipitating factors, including poorly controlled diabetes mellitus, alcohol consumption, or therapy with certain medications, are also implicated in the pathogenesis of HTGP. Similarly, genetic factors may also be deemed responsible for altering the normal lipid metabolism in these patients [6].

The overall mortality following acute pancreatitis has been described up to $5 \%$; however, it is relatively higher among hospitalized patients [6]. HTGP frequently culminates in a severe disease presentation with an increased propensity to cause life-threatening complications. In this context, an effective and feasible treatment of choice is particularly warranted. The major goal of therapy is to relieve pain and avoid any inadvertent events. In the current times, a specific therapeutic approach is non-existent. Conservative management, using the variable combinations of intravenous hydration, analgesics, and antibiotics, is important for the initial resuscitation. Although heparin and insulin have been used, plasmapheresis and lipid apheresis are becoming more popular management options for hyperlipidemic pancreatitis [7]. However, the widespread availability of these techniques may pose a therapeutic dilemma. This review outlines our current understanding of the epidemiology and risk factors for HTGP, the pathophysiology of this disorder, and currently available approaches to diagnosis and treatment. We highlight insulin therapy as a feasible therapeutic option in these patients, especially if plasmapheresis is not available.

\section{Review}

\section{Epidemiology}

HTGP is a known clinical entity with an estimated incidence ranging from $2.3 \%$ to $10 \%$ of all cases of acute pancreatitis [7]. It often involves male individuals with age less than 50 years who have severe 
hypertriglyceridemia. According to a prospective study of 400 cases of acute pancreatitis, patients with hypertriglyceridemia were younger, obese, predominantly of the male gender, diabetic, and had a history of more frequent episodes of persistent organ failure [8]. Furthermore, hypertriglyceridemia is commonly encountered in pregnancy due to physiological changes and, occasionally, it may lead to acute pancreatitis. The incidence of HTGP during pregnancy has been described to be 1 in 25,000 pregnancies.

\section{Pathogenesis}

Although the exact pathogenesis of HTGP is unknown, the proposed mechanism implicates the toxic effects of free fatty acids released by the breakdown of triglycerides [9]. Triglyceride-rich lipoproteins such as chylomicrons and very low-density lipoproteins (VLDLs) are large molecules that are found in abundance in conditions like hypertriglyceridemia. These molecules may occlude the pancreatic capillaries and subsequently change the structure of the acinar cells. This process eventually triggers the release of pancreatic enzymes, including lipases that catabolize the lipid-rich molecules. This breakdown results in increased local oxidative stress that further contributes to the inflammatory response in the pancreas, resulting in the symptomatology of acute pancreatitis [9].

\section{Search criteria}

We conducted an extensive literature search of Google Scholar and PubMed (National Library of Medicine, Bethesda, MD) using a combination of terms, including "hypertriglyceridemia," "pancreatitis," "insulin," and "treatment." A total of 147 studies were initially obtained, consisting of but not limited to original articles, case series, and case reports as of July 2018. After excluding 39 duplicate articles, 108 papers were thoroughly studied. The articles available in any language other than the English were excluded from the review. Forty-eight papers were found relevant to the scope of our study but 34 cases were found accessible in order to retrieve pertinent data required for this review [10-31]. The data on individual cases of HTGP on epidemiology, clinical presentation, diagnosis, management, and clinical outcomes are summarized in Table 1 .

\begin{tabular}{|c|c|c|c|c|c|c|c|c|}
\hline Authors & Age/Gender & $\begin{array}{l}\text { Clinical } \\
\text { presentation }\end{array}$ & Comorbidity & $\begin{array}{l}\text { Initial } \\
\text { TG } \\
\text { level } \\
\text { (mg/dL) }\end{array}$ & Treatment & $\begin{array}{l}\text { TG post- } \\
\text { treatment } \\
\text { (mg/dL) }\end{array}$ & $\begin{array}{l}\text { Duration } \\
\text { of } \\
\text { treatment } \\
\text { (days) }\end{array}$ & Outcome \\
\hline $\begin{array}{l}\text { Jabbar et } \\
\text { al. } 1998 \\
{[10]}\end{array}$ & 13/M & Abdominal pain & HTG & 4574 & $\begin{array}{l}\text { Regular insulin injection } \\
(0.1 \mathrm{U} / \mathrm{kg} / \mathrm{h}) \text {, fish oil, } \\
\text { gemfibrozil }\end{array}$ & $565^{\star}$ & 1 & Recovered \\
\hline $\begin{array}{l}\text { Huang et } \\
\text { al. } 2002 \\
{[11]}\end{array}$ & 41/F & $\begin{array}{l}\text { Abdominal pain, } \\
\text { nausea, vomiting }\end{array}$ & HTG, OCPs & 2260 & Insulin-glucose infusion & 314 & 3 & Recovered \\
\hline $\begin{array}{l}\text { Mikhail et } \\
\text { al. } 2005 \\
{[12]}\end{array}$ & $38 / F$ & $\begin{array}{l}\text { Excruciating } \\
\text { abdominal pain }\end{array}$ & HTG & 10,560 & $\begin{array}{l}\text { Subcutaneous insulin } \\
\text { lispro, sliding scale }\end{array}$ & 712 & 3 & Recovered \\
\hline $\begin{array}{l}\text { Alagozlu } \\
\text { et al. } 2006 \\
\text { [13] }\end{array}$ & 44/M & $\begin{array}{l}\text { Abdominal pain, } \\
\text { vomiting }\end{array}$ & DM type 2 & 1707 & $\begin{array}{l}\text { Regular insulin } 3 \mathrm{U} / \mathrm{h} \text {, } \\
\mathrm{D} 5 \mathrm{~W}, 5000 \mathrm{U} \text { of heparin }\end{array}$ & 180 & 10 & Recovered \\
\hline $\begin{array}{l}\text { Gursoy et } \\
\text { al. } 2006 \\
{[14]}\end{array}$ & $24 / F$ & $\begin{array}{l}\text { Severe abdominal } \\
\text { pain, fever, } \\
\text { vomiting, malaise }\end{array}$ & Pregnancy & 10,092 & $\begin{array}{l}\text { Intravenous insulin, } \\
\text { glucose }\end{array}$ & 608 & 5 & Recovered \\
\hline $\begin{array}{l}\text { Jain et al. } \\
2007 \text { [15] }\end{array}$ & 55/M & $\begin{array}{l}\text { Abdominal pain, } \\
\text { vomiting }\end{array}$ & $\begin{array}{l}\text { Recurrent acute } \\
\text { pancreatitis }\end{array}$ & 1808 & $\begin{array}{l}\text { Regular insulin } \\
\text { infusion, D5W, } 5000 \mathrm{U} \\
\text { heparin subcutaneously } \\
\text { twice a day }\end{array}$ & 325 & 5 & Recovered \\
\hline $\begin{array}{l}\text { Jain et al. } \\
2007 \text { [15] }\end{array}$ & $46 / \mathrm{M}$ & $\begin{array}{l}\text { Abdominal pain, } \\
\text { vomiting }\end{array}$ & $\begin{array}{l}\text { DM, recurrent } \\
\text { pancreatitis }\end{array}$ & 3743 & $\begin{array}{l}\text { Regular insulin } \\
\text { infusion, D5W, } 5000 \mathrm{U} \\
\text { heparin subcutaneously } \\
\text { twice a day }\end{array}$ & 350 & 5 & Recovered \\
\hline $\begin{array}{l}\text { Love et al. } \\
2009 \text { [16] }\end{array}$ & $34 / F$ & $\begin{array}{l}\text { Abdominal pain, } \\
\text { nausea, vomiting, } \\
\text { diarrhea }\end{array}$ & $\begin{array}{l}\text { HTN, HLD, CD, } \\
\text { cholecystectomy }\end{array}$ & 10,039 & $\begin{array}{l}\text { Sliding scale insulin, } \\
\text { gemifibrozil }\end{array}$ & 646 & 9 & Recovered \\
\hline Jain et al. & 54/M & Acute epigastric & DM, HTG & 10,320 & Regular insulin & 1386 & 6 & Recovered \\
\hline
\end{tabular}




\section{Cureus}

\begin{tabular}{|c|c|c|c|c|c|c|c|c|}
\hline 2009 [17] & & pain & & & infusion, D5W, heparin & & & \\
\hline $\begin{array}{l}\text { Hahn et al. } \\
2010 \text { [18] }\end{array}$ & $20 / F$ & $\begin{array}{l}\text { Epigastric pain, } \\
\text { vomiting, } \\
\text { diarrhea }\end{array}$ & DM type 1 & 15,240 & $\begin{array}{l}\text { Continuous insulin } \\
\text { infusion, } 3 \% \mathrm{NaCl} \\
\text { solution for } \\
\text { pseudohyponatremia }\end{array}$ & 309 & 22 & Recovered \\
\hline $\begin{array}{l}\text { Twilla et } \\
\text { al. } 2012 \\
\text { [19] }\end{array}$ & $39 / \mathrm{M}$ & $\begin{array}{l}\text { Abdominal pain, } \\
\text { nausea, and } \\
\text { vomiting }\end{array}$ & HTG & 5366 & $\begin{array}{l}\text { Continuous insulin } \\
\text { infusion and } \\
\text { subcutaneous heparin }\end{array}$ & 717 & 5 & Recovered \\
\hline $\begin{array}{l}\text { Patel et al. } \\
2012 \text { [20] }\end{array}$ & $42 / \mathrm{F}$ & $\begin{array}{l}\text { Abdominal pain, } \\
\text { vomiting }\end{array}$ & $\begin{array}{l}\text { DM type } 2 \\
\text { type } 5 \text { HTG }\end{array}$ & $>5000$ & $\begin{array}{l}\text { Insulin infusion } 0.1 \\
\mathrm{U} / \mathrm{kg} / \mathrm{h} \text {, D5W, heparin } 600 \\
\mathrm{U} / \mathrm{h}\end{array}$ & 923 & 1 & Recovered \\
\hline $\begin{array}{l}\text { Weston et } \\
\text { al. 2013 } \\
\text { [21] }\end{array}$ & 46/M & $\begin{array}{l}\text { Abdominal pain, } \\
\text { eruptive } \\
\text { xanthomas arms } \\
\text { and torso }\end{array}$ & $\begin{array}{l}2 \text { Mls status } \\
\text { post } C A B G \\
\text { surgery and } \\
\text { stenting }\end{array}$ & 3026 & Insulin-heparin infusion & NA & NA & Recovered \\
\hline $\begin{array}{l}\text { Denecker } \\
\text { et al. } 2013 \\
\text { [22] }\end{array}$ & $23 / \mathrm{F}$ & $\begin{array}{l}\text { Epigastric pain, } \\
\text { nausea }\end{array}$ & DM type 2, RE & 12,851 & $\begin{array}{l}\text { Intravenous fluid, insulin, } \\
\text { analgesics }\end{array}$ & 549 & 12 & Recovered \\
\hline $\begin{array}{l}\text { Coskun et } \\
\text { al. } 2015 \\
\text { [23] }\end{array}$ & 41/M & $\begin{array}{l}\text { Abdominal pain, } \\
\text { nausea, vomiting }\end{array}$ & HTG & 1118 & $\begin{array}{l}\text { Regular insulin infusion, } \\
\text { D5W }\end{array}$ & 355 & 3 & Recovered \\
\hline $\begin{array}{l}\text { Coskun et } \\
\text { al. } 2015 \\
{[23]}\end{array}$ & $48 / \mathrm{F}$ & $\begin{array}{l}\text { Abdominal pain, } \\
\text { nausea, vomiting }\end{array}$ & DM & 1176 & $\begin{array}{l}\text { Regular insulin infusion, } \\
\text { D5W }\end{array}$ & 464 & 3 & Recovered \\
\hline $\begin{array}{l}\text { Coskun et } \\
\text { al. } 2015 \\
{[23]}\end{array}$ & $54 / \mathrm{M}$ & $\begin{array}{l}\text { Abdominal pain, } \\
\text { nausea, vomiting }\end{array}$ & HTG & 1228 & $\begin{array}{l}\text { Intravenous regular } \\
\text { insulin infusion in D5W }\end{array}$ & 489 & 3 & Recovered \\
\hline $\begin{array}{l}\text { Coskun et } \\
\text { al. } 2015 \\
\text { [23] }\end{array}$ & $35 / \mathrm{M}$ & $\begin{array}{l}\text { Abdominal pain, } \\
\text { vomiting }\end{array}$ & DM & 1027 & $\begin{array}{l}\text { Regular insulin infusion, } \\
\text { D5W }\end{array}$ & 496 & 3 & Recovered \\
\hline $\begin{array}{l}\text { Coskun et } \\
\text { al. } 2015 \\
\text { [23] }\end{array}$ & $43 / F$ & $\begin{array}{l}\text { Abdominal pain, } \\
\text { nausea, vomiting }\end{array}$ & HTG & 1004 & $\begin{array}{l}\text { Intravenous regular } \\
\text { insulin infusion in D5W }\end{array}$ & 476 & 3 & Recovered \\
\hline $\begin{array}{l}\text { Coskun et } \\
\text { al. } 2015 \\
\text { [23] }\end{array}$ & $30 / F$ & $\begin{array}{l}\text { Abdominal pain, } \\
\text { nausea, vomiting }\end{array}$ & HTG & 1086 & $\begin{array}{l}\text { Intravenous regular } \\
\text { insulin infusion in D5W }\end{array}$ & 481 & 3 & Recovered \\
\hline $\begin{array}{l}\text { Coskun et } \\
\text { al. } 2015 \\
\text { [23] }\end{array}$ & 59/M & $\begin{array}{l}\text { Abdominal pain, } \\
\text { nausea, vomiting }\end{array}$ & DM & 1130 & $\begin{array}{l}\text { Regular insulin infusion, } \\
\text { D5W }\end{array}$ & 373 & 5 & Recovered \\
\hline $\begin{array}{l}\text { Coskun et } \\
\text { al. } 2015 \\
\text { [23] }\end{array}$ & 46/M & $\begin{array}{l}\text { Abdominal pain, } \\
\text { nausea, vomiting }\end{array}$ & DM & 1156 & $\begin{array}{l}\text { Intravenous regular } \\
\text { insulin infusion in D5W }\end{array}$ & 498 & 3 & Recovered \\
\hline $\begin{array}{l}\text { Coskun et } \\
\text { al. } 2015 \\
\text { [23] }\end{array}$ & $40 / F$ & $\begin{array}{l}\text { Abdominal pain, } \\
\text { vomiting }\end{array}$ & DM & 1124 & $\begin{array}{l}\text { Regular insulin infusion, } \\
\text { D5W }\end{array}$ & 494 & 3 & Recovered \\
\hline $\begin{array}{l}\text { Coskun et } \\
\text { al. } 2015 \\
{[23]}\end{array}$ & 45/M & $\begin{array}{l}\text { Abdominal pain, } \\
\text { nausea, vomiting }\end{array}$ & DM & 1235 & $\begin{array}{l}\text { Intravenous regular } \\
\text { insulin infusion in D5W }\end{array}$ & 276 & 5 & Recovered \\
\hline $\begin{array}{l}\text { Coskun et } \\
\text { al. } 2015 \\
\text { [23] }\end{array}$ & $65 / \mathrm{M}$ & $\begin{array}{l}\text { Abdominal pain, } \\
\text { vomiting }\end{array}$ & DM & 1190 & $\begin{array}{l}\text { Intravenous regular } \\
\text { insulin infusion in D5W }\end{array}$ & 356 & 5 & Recovered \\
\hline $\begin{array}{l}\text { Coskun et } \\
\text { al. } 2015 \\
\text { [23] }\end{array}$ & 46/M & $\begin{array}{l}\text { Abdominal pain, } \\
\text { nausea, vomiting }\end{array}$ & DM & 1215 & $\begin{array}{l}\text { Regular insulin infusion, } \\
\text { D5W }\end{array}$ & 298 & 5 & Recovered \\
\hline
\end{tabular}




\section{Cureus}

\begin{tabular}{|c|c|c|c|c|c|c|c|c|}
\hline $\begin{array}{l}\text { Franco et } \\
\text { al. } 2015 \\
{[24]}\end{array}$ & 50/M & $\begin{array}{l}\text { Severe epigastric } \\
\text { pain }\end{array}$ & $\begin{array}{l}\text { BD, HLD, DM } \\
\text { type 2, obesity, } \\
\text { IBS, GAD }\end{array}$ & 3590 & Insulin infusion & 684 & 3 & Recovered \\
\hline $\begin{array}{l}\text { Khan et al. } \\
2015 \text { [25] }\end{array}$ & $44 / F$ & $\begin{array}{l}\text { Abdominal pain, } \\
\text { vomiting }\end{array}$ & HTN & 3525 & Insulin infusion & 675 & 3 & Recovered \\
\hline $\begin{array}{l}\text { Singla et } \\
\text { al. } 2015 \\
{[26]}\end{array}$ & 19/M & $\begin{array}{l}\text { Epigastric pain, } \\
\text { nausea, polyuria }\end{array}$ & Obesity, DM & 4009 & $\begin{array}{l}\text { Insulin infusion } 6 \mathrm{U} / \mathrm{h} \text {, } \\
\text { D5W, fenofibrate }\end{array}$ & 180 & 1 & Recovered \\
\hline $\begin{array}{l}\text { Amin et al. } \\
2015 \text { [27] }\end{array}$ & $40 / F$ & $\begin{array}{l}\text { Abdominal pain, } \\
\text { vomiting, } \\
\text { diarrhea }\end{array}$ & $\begin{array}{l}\text { Graves diseases, } \\
\text { HTG, pregnancy }\end{array}$ & 4106 & $\begin{array}{l}\text { Low-dose insulin on } \\
\text { sliding scale }\end{array}$ & 885 & 8 & Recovered \\
\hline $\begin{array}{l}\text { Abraham } \\
\text { et al. } 2015 \\
\text { [28] }\end{array}$ & $24 / F$ & $\begin{array}{l}\text { Abdominal pain, } \\
\text { nausea, vomiting }\end{array}$ & $\begin{array}{l}\text { Asthma, OCP } \\
\text { Estrostep }\end{array}$ & 2200 & $\begin{array}{l}\text { Insulin infusion, omega-3 } \\
\text { fatty acids, gemifibrozil }\end{array}$ & 355 & 7 & Recovered \\
\hline $\begin{array}{l}\text { Aryal et al. } \\
2016 \text { [29] }\end{array}$ & $31 / \mathrm{M}$ & Epigastric pain & $\begin{array}{l}\text { Obesity, HLD, } \\
\text { HTN, DM type } 2\end{array}$ & 15,215 & $\begin{array}{l}\text { Regular insulin ( } 0.4 \\
\mathrm{U} / \mathrm{kg} / \mathrm{h} \text { ), heparin infusion }\end{array}$ & 363 & 6 & Recovered \\
\hline $\begin{array}{l}\text { Jeon et al. } \\
2017 \text { [30] }\end{array}$ & $28 / F$ & Epigastric pain & HLD, Pregnancy & 10,392 & Insulin injection & $\begin{array}{l}\text { No } \\
\text { improvement }\end{array}$ & NA & $\begin{array}{l}\text { Died } \\
\text { (cardiac } \\
\text { arrest after } \\
\text { AP-related } \\
\text { DKA) }\end{array}$ \\
\hline $\begin{array}{l}\text { Chaudhary } \\
\text { et al. } 2017 \\
\text { [31] }\end{array}$ & $44 / \mathrm{M}$ & $\begin{array}{l}\text { Epigastric } \\
\text { pain, vomiting }\end{array}$ & $\begin{array}{l}\text { DM, HTG, HTN, } \\
\text { obesity, OSA }\end{array}$ & 6,672 & $\begin{array}{l}\text { Insulin infusion } 0.1 \\
\mathrm{u} / \mathrm{kg} / \mathrm{h} \text {, D5W } 75 \mathrm{cc} / \mathrm{h}\end{array}$ & 500 & 8 & Recovered \\
\hline
\end{tabular}

\section{TABLE 1: Literature review of insulin treatment in patients with hypertriglyceridemia-associated acute pancreatitis}

TG, triglyceride; HTG, hypertriglyceridemia; OCPs, oral contraceptive pills; DM, diabetes mellitus; U, unit; D5W, dextrose 5\% in water; HTN, hypertension; HLD, hyperlipidemia; CD, Crohn's disease; MI, myocardial infarction; CABG, coronary artery bypass graft; RE, reflux esophagitis; BD, bipolar disorder; IBS; irritable bowel syndrome; GAD, generalized anxiety disorder; AP; acute pancreatitis; DKA; diabetic ketoacidosis; OSA, obstructive sleep apnea; NA, not available

*After acute phase of insulin treatment

\section{Patient demographics and clinical presentations}

In the present review, HTGP involved all age groups, with the mean age of 39.6 years (range: 13-65 years). There was no clear gender preponderance (male, $\mathrm{n}=18$; female, $\mathrm{n}=16$ ). The typical presentation was acuteonset abdominal pain while other notable symptoms included nausea and vomiting. Cutaneous signs of hypertriglyceridemia such as eruptive xanthomas over the extensor surfaces of the arms, legs, and buttocks were also noted in a few patients. A majority of patients had comorbid conditions and risk factors like moderate-to-severe hypertriglyceridemia, hypertension, diabetes mellitus, dyslipidemias, pregnancy, alcohol abuse, oral contraceptive use, and obesity. It was an interesting observation that the initial triglyceride levels causing acute pancreatitis were more than $1000 \mathrm{mg} / \mathrm{dL}$ in all cases included in this review. It was further notable that there were a few cases where HTGP occurred in pregnant patients. Although pregnancy is associated with an overall increase in the serum triglyceride level, it rarely exceeds $300 \mathrm{mg} / \mathrm{dL}$ in most pregnant females.

\section{Diagnosis}

The diagnostic criteria for HTGP include the presence of at least two out of three following findings: (a) acute-onset severe epigastric pain radiating to the back; (b) serum lipase or amylase elevated three or more times the upper reference limit; and (c) the three characteristic findings of acute pancreatitis on imaging investigations such as computed tomography, magnetic resonance imaging, or transabdominal ultrasonography [32]. Another major clue for the diagnosis of HTGP includes biochemical evaluation remarkable for serum triglyceride levels more than $1000 \mathrm{mg} / \mathrm{dL}$. The risk factors for hypertriglyceridemia are obesity, pregnancy, family history of hypertriglyceridemia, poorly-controlled diabetes mellitus, and alcoholism [32]. It is important to mention here that a thorough clinical history, including family history of lipid metabolic aberrations and physical examination to identify eruptive xanthomas, can help to channelize the biochemical and radiological investigations toward a timely etiology establishment. 


\section{Insulin therapy}

Currently, there are no clear therapeutic guidelines for HTGP. Insulin therapy has previously been used in these patients as a minimally invasive and economical strategy with promising outcomes [33-34]. The mechanism by which insulin lowers the level of serum triglycerides is by triggering the enzymatic activity of lipoprotein lipase and inhibition of hormone-sensitive lipase. Lipoprotein lipase metabolizes chylomicrons and VLDLs into the free fatty acids and glycerol. Therefore, it ultimately decreases the serum triglyceride levels. Decreasing the activity of hormone-sensitive lipase causes decreased adipocyte-triglyceride breakdown, resulting in a decreased release of free fatty acids into the circulation, which controls the toxic effects on the pancreas, limiting its active inflammation [35]. In this review, the initial symptomatic management comprised of bowel rest, intravenous fluids, and analgesics. In regard to the emergency management of HTGP, insulin infusion as monotherapy or part of a combination regimen was the most effective option in settings where plasmapheresis was not available or as an alternative approach for patients who could not tolerate apheresis. It was usually given intravenously at a rate of $0.1-0.3$ units $/ \mathrm{kg} / \mathrm{hour}$. Serum triglyceride levels were monitored every 12 hours. With insulin therapy, it was pivotal to measure blood glucose levels and adjuvant $5 \%$ dextrose infusion was required when the blood glucose level fell below 200 $\mathrm{mg} / \mathrm{dL}$.

\section{Clinical outcomes}

According to the results of the outcome analysis of this review, the overall prognosis of HTGP was good with a vast majority of patients recovering completely with intensive insulin therapy. It is quite reassuring that in emergency clinical settings, intravenous insulin was used as a salvage therapy, even in patients with severe HTGP, resulting in a remarkable recovery. In three to five days of insulin therapy, most patients demonstrated a good clinical response; serum triglyceride levels decreased to less than $500 \mathrm{mg} / \mathrm{dL}$ after which the treatment was discontinued. A number of patients received insulin as a combination therapy with lipid-lowering drugs. In our review, one patient died from HTGP due to cardiac arrest, which was thought to occur as a sequel to severe metabolic acidosis and electrolyte imbalance. This fatal outcome re-emphasizes that patients with HTGP are more likely to encounter organ failure as compared to the other causes of pancreatitis. Therefore, urgent and appropriate management is essentially important.

\section{Long-term management}

Patients with HTGP clinically improve when the serum triglyceride levels fall below $500 \mathrm{mg} / \mathrm{dL}$. However, in order to prevent recurrent episodes and subsequent complications of HTGP, long-term management is warranted to maintain the level of triglycerides below $200 \mathrm{mg} / \mathrm{dL}$. It is particularly tailored to lifestyle modifications with dietary fat and sugar restriction, aerobic exercises, weight loss, and blood sugar control. The hypolipidemic medications like gemfibrozil and fenofibrate lower serum triglyceride levels and reduce the recurrence risk of HTGP [36-37].

\section{Future directions}

A prospective randomized controlled trial showed that early high-volume hemofiltration can lower triglyceride levels more efficiently than low-molecular-weight heparin combined with insulin therapy, but it was not superior in terms of clinical outcomes and cost [38]. Therefore, the determination of relative efficacy, meticulous risk stratification, and mortality benefits of currently used techniques, that is, insulin therapy and plasmapheresis warrant further research. Randomized clinical trials in the future may help untangle this uncertainty about the best technique in regards to the emergency management of HTGP.

\section{Conclusions}

Patients with HTGP require urgent management as the disease presentation is particularly severe and it may result in grave complications. The use of insulin therapy with close monitoring of blood glucose levels can be an appropriate therapeutic approach, especially in cases with no availability of apheresis. This paper not only highlights the utility of insulin therapy for HTGP but also sensitizes concerned physicians to evaluate this treatment approach in larger, multicenter studies. Long-term management using pharmacological and non-pharmacological therapies, directed at maintaining the serum triglycerides within normal limits, is required to prevent recurrent attacks of HTGP.

\section{Additional Information \\ Disclosures}

Conflicts of interest: In compliance with the ICMJE uniform disclosure form, all authors declare the following: Payment/services info: All authors have declared that no financial support was received from any organization for the submitted work. Financial relationships: All authors have declared that they have no financial relationships at present or within the previous three years with any organizations that might have an interest in the submitted work. Other relationships: All authors have declared that there are no other relationships or activities that could appear to have influenced the submitted work. 


\section{References}

1. de Pretis N, Amodio A, Frulloni L: Hypertriglyceridemic pancreatitis: epidemiology, pathophysiology and clinical management. United European Gastroenterol J. 2018, 6:649-655. 10.1177/2050640618755002

2. Inayat F, Virk HU, Yoon DJ, Riaz I: Drug-induced pancreatitis: a rare manifestation of doxycycline administration. N Am J Med Sci. 2016, 8:117-120. 10.4103/1947-2714.174348

3. Inayat F, Virk HU, Cheema AR, Saif MW: Plasmablastic lymphoma mimicking acute pancreatitis. Case Rep Oncol Med. 2016, 2016:9751736. 10.1155/2016/9751736

4. Tsuang W, Navaneethan U, Ruiz L, Palascak JB, Gelrud A: Hypertriglyceridemic pancreatitis: presentation and management. Am J Gastroenterol. 2009, 104:984-991. 10.1038/ajg.2009.27

5. Lithell H, Vessby B, Walldius G, Carlson LA: Hypertriglyceridemia-acute pancreatitis-ischemic heart disease. a case study in a pair of monozygotic twins. Acta Med Scand. 1987, 221:311-316. 10.1111/j.09546820.1987.tb00900.x

6. Cavallini G, Frulloni L, Bassi C, et al.: Prospective multicentre survey on acute pancreatitis in Italy (ProInfAISP): results on 1005 patients. Dig Liver Dis. 2004, 36:205-211. 10.1016/j.dld.2003.11.027

7. Yeh JH, Chen JH, Chiu HC: Plasmapheresis for hyperlipidemic pancreatitis. J Clin Apher. 2003, 18:181-185. 10.1002/jca.10063

8. Nawaz H, Koutroumpakis E, Easler J, et al.: Elevated serum triglycerides are independently associated with persistent organ failure in acute pancreatitis. Am J Gastroenterol. 2015, 110:1497-1503. 10.1038/ajg.2015.261

9. Navina S, Acharya C, DeLany JP, et al.: Lipotoxicity causes multisystem organ failure and exacerbates acute pancreatitis in obesity. Sci Transl Med. 2011, 3:107-110. 10.1126/scitranslmed.3002573

10. Jabbar MA, Zuhri-Yafi MI, Larrea J: Insulin therapy for a non-diabetic patient with severe hypertriglyceridemia. J Am Coll Nutr. 1998, 17:458-461. 10.1080/07315724.1998.10718794

11. Huang DB, Raskin P: Diabetic hypertriglyceridemia-induced acute pancreatitis masquerading as biliary pancreatitis. J Diabetes Complications. 2002, 16:180-182. 10.1016/S1056-8727(01)00183-0

12. Mikhail N, Trivedi K, Page C, Wali S, Cope D: Treatment of severe hypertriglyceridemia in nondiabetic patients with insulin. Am J Emerg Med. 2005, 23:415-417. 10.1016/j.ajem.2005.02.036

13. Alagozlu H, Cindoruk M, Karakan T, Unal S: Heparin and insulin in the treatment of hypertriglyceridemiainduced severe acute pancreatitis. Dig Dis Sci. 2006, 51:931-933. 10.1007/s10620-005-9006-Z

14. Gursoy A, Kulaksizoglu M, Sahin M, Ertugrul DT, Ozer F, Tutuncu NB, Demirag NG: Severe hypertriglyceridemia-induced pancreatitis during pregnancy. J Natl Med Assoc. 2006, 98:655-657.

15. Jain P, Rai RR, Udawat H, Nijhawan S, Mathur A: Insulin and heparin in treatment of hypertriglyceridemiainduced pancreatitis. World J Gastroenterol. 2007, 13:2642-2643. 10.3748/wig.v13.i18.2642

16. Love BL, Kehr H, Olin JL: Hypertriglyceridaemia-induced acute pancreatitis due to patient non-compliance. J Clin Pharm Ther. 2009, 34:363-367. 10.1111/j.1365-2710.2008.01002.x

17. Jain D, Zimmerschied J: Heparin and insulin for hypertriglyceridemia-induced pancreatitis: case report. ScientificWorldJournal. 2009, 9:1230-1232. 10.1100/tsw.2009.142

18. Hahn SJ, Park JH, Lee JH, Lee JK, Kim KA: Severe hypertriglyceridemia in diabetic ketoacidosis accompanied by acute pancreatitis: case report. J Korean Med Sci. 2010, 25:1375-1378. 10.3346/jkms.2010.25.9.1375

19. Twilla JD, Mancell J: Hypertriglyceridemia-induced acute pancreatitis treated with insulin and heparin . Am J Health Syst Pharm. 2012, 69:213-216. 10.2146/ajhp110144

20. Patel AD: Hypertriglyceridemia-induced acute pancreatitis treatment with insutin and heparin . Indian J Endocrinol Metab. 2012, 16:671-672. 10.4103/2230-8210.98050

21. Weston N, Fernando U, Baskar V: Hypertriglyceridaemia-induced pancreatitis. BMJ Case Rep. 2013, 2013:2013008722. 10.1136/bcr-2013-008722

22. Denecker N, Decochez K: Poorly controlled type 2 diabetes complicated by an episode of severe hypertriglyceridaemia-induced pancreatitis. BMJ Case Rep. 2013, 2013:2012008455. 10.1136/bcr-2012008455

23. Coskun A, Erkan N, Yakan S, Yildirim M, Carti E, Ucar D, Oymaci E: Treatment of hypertriglyceridemiainduced acute pancreatitis with insulin. Prz Gastroenterol. 2015, 10:18-22. 10.5114/pg.2014.45412

24. Franco JM, Vallabhajosyula S, Griffin TJ: Quetiapine-induced hypertriglyceridaemia causing acute pancreatitis. BMJ Case Rep. 2015, 2015:2015209571. 10.1136/bcr-2015-209571

25. Khan R, Jehangir W, Regeti K, Yousif A: Hypertriglyceridemia-induced pancreatitis: choice of treatment Gastroenterology Res. 2015, 8:234-236. 10.14740/gr662e

26. Singla AA, Ting F, Singla A: Acute pancreatitis secondary to diabetic ketoacidosis induced hypertriglyceridemia in a young adult with undiagnosed type 2 diabetes. JOP. 2015, 16:201-204. 10.6092/1590-8577/2961

27. Amin T, Poon LC, Teoh TG, Moorthy K, Robinson S, Neary N, Valabhji J: Management of hypertriglyceridaemia-induced acute pancreatitis in pregnancy. J Matern Fetal Neonatal Med. 2015, 28:954958. 10.3109/14767058.2014.939064

28. Abraham M, Mitchell J, Simsovits D, Gasperino J: Hypertriglyceridemic pancreatitis caused by the oral contraceptive agent estrostep. J Intensive Care Med. 2015, 30:303-307. 10.1177/0885066614528083

29. Aryal MR, Mainali NR, Gupta S, Singla M: Acute pancreatitis owing to very high triglyceride levels treated with insulin and heparin infusion. BMJ Case Rep. 2013, 2013:2013008550. 10.1136/bcr-2013-008550

30. Jeon HR, Kim SY, Cho YJ, Chon SJ: Hypertriglyceridemia-induced acute pancreatitis in pregnancy causing maternal death. Obstet Gynecol Sci. 2016, 59:148-151. 10.5468/ogs.2016.59.2.148

31. Chaudhary A, Iqbal U, Anwar H, Siddiqui HU, Alvi M: Acute pancreatitis secondary to severe hypertriglyceridemia: management of severe hypertriglyceridemia in emergency setting. Gastroenterology Res. 2017, 10:190-192. 10.14740/gr762e

32. Forsmark CE, Baillie J, AGA Institute Clinical Practice and Economics Committee, AGA Institute Governing Board: AGA Institute technical review on acute pancreatitis . Gastroenterology. 2007, 132:2022-2044. 10.1053/j.gastro.2007.03.065

33. Rawla P, Sunkara T, Thandra KC, Gaduputi V: Hypertriglyceridemia-induced pancreatitis: updated review of 


\section{Cureus}

current treatment and preventive strategies. Clin J Gastroenterol. 2018, 1-8. 10.1007/s12328-018-0881-1

34. Inayat F, Zafar F, Riaz I, Younus F, Baig AS, Imran Z: Hypertriglyceridemic pancreatitis: is insulin monotherapy a feasible therapeutic option?. Cureus. 2018, 10:3461. 10.7759/cureus.3461

35. Eckel RH: Lipoprotein lipase. a multifunctional enzyme relevant to common metabolic diseases . N Engl J Med. 1989, 320:1060-1068. 10.1056/NEJM198904203201607

36. Lim R, Rodger SJ, Hawkins TL: Presentation and management of acute hypertriglyceridemic pancreatitis in pregnancy: a case report. Obstet Med. 2015, 8:200-203. 10.1177/1753495X15605697

37. Preiss D, Tikkanen MJ, Welsh P, et al.: Lipid-modifying therapies and risk of pancreatitis: a meta-analysis . JAMA. 2012, 308:804-811. 10.1001/jama.2012.8439

38. He WH, Yu M, Zhu Y, et al.: Emergent triglyceride-lowering therapy with early high-volume hemofiltration against low-molecular-weight heparin combined with insulin in hypertriglyceridemic pancreatitis: a prospective randomized controlled trial. J Clin Gastroenterol. 2016, 50:772-778.

10.1097/MCG.0000000000000552 